\title{
Mortality in Fetal Alcohol Spectrum Disorders
}

\author{
Alex Thompson', Dawn Hackman², Larry Burd1 \\ ${ }^{1}$ Department of Pediatrics, University of North Dakota School of Medicine and Health Sciences, Grand Forks, \\ USA \\ ${ }^{2}$ Harley E. French Library of the Health Sciences, University of North Dakota School of Medicine and Health \\ Sciences, Grand Forks, USA \\ Email: larry.burd@med.und.edu
}

Received 28 November 2013; revised 25 December 2013; accepted 3 January 2014

Copyright (C) 2014 by authors and Scientific Research Publishing Inc.

This work is licensed under the Creative Commons Attribution International License (CC BY).

http://creativecommons.org/licenses/by/4.0/

\section{(c) (i) Open Access}

\section{Abstract}

Objective: Mortality in FASD has not been well studied. In this paper we review published reports of mortality in FASD. Method: We searched using Pub Med for all years in all languages for reports of all-cause mortality associated with any FASD. Results: We located 26 papers reporting on 57 deaths. Cause of death was reported for $49 / 57$ cases (86\%). The two most prevalent potential causes of death were malformations of the heart ( 37 of 49 cases, $75.5 \%$ ) which varied from atrial septal defect or patent ductus arteriosus to tetralogy of Fallot, hypoplastic left heart, aortic arch interruption, etc. and brain malformations ( 25 of $49,51 \%)$ including microcephaly, hydrocephalus, porencephaly, agenesis/absence of the corpus callosum and semilobar holoprosencephaly. In several cases potential causal findings overlapped. The three most frequent other causes of death were sepsis ( 7 cases, $14.3 \%$ ), kidney malformations ( 7 cases, $14.3 \%$ ), and cancer ( 4 cases, $8.2 \%$ ). Over half the deaths $(30 / 55,54.5 \%)$ occurred in the first year of life. Discussion: We found that congenital heart disease was the most common cause of death in people with FASD. This may be due to an ascertainment bias since many of the published studies were focused on congenital heart disease in FASD. We conclude that FASD is frequently undetected in mortality events and could be a common finding in infant, child, adolescent and adult mortality.

\section{Keywords}

Fetal Alcohol Spectrum Disorder; Mortality; Birth Defects; Heart Defects; Brain Malformations; Sepsis; Cancer

\section{Introduction}

In 1899, a prison medical officer reported on the role of maternal alcoholism in infant mortality [1]. The rate of 
infant mortality was doubled in alcoholic mothers. Later-born children had increased risk compared to earlier births (1st-born, 33.7\%; 6th- to 10th-born, 72\%). In the 299 surviving children born to mothers with a previous child death, the rate of epilepsy was $4.1 \%$ (10 - 40 times higher than rates reported in the general population at that time). One of the first descriptions of outcomes in children of alcohol-exposed pregnancies was published in 1968 [2]. They reported increased rates of miscarriages, stillbirths, prematurity and birth defects.

World-wide alcohol abuse now accounts for $4 \%$ of total worldwide mortality and between $4 \%$ and $5 \%$ of all disability-adjusted life years [3]. The prevalence of drinking during pregnancy is quite high especially prior to pregnancy recognition when slightly more than $50 \%$ of women in the United States report some alcohol use [4] [5]. Since many pregnancies are unplanned, early exposure is common. The prevalence of alcohol exposure decreases from $50 \%$ prior to pregnancy recognition to around $10 \%$ upon recognition of pregnancy [5]-[7]. About 3\% - 5\% of women continue to drink throughout pregnancy and many drink heavily, more than five drinks per occasion and often several times per week [4]. Thus, several hundred thousand pregnancies have prenatal alco- hol exposure at levels increasing risk for a fetal alcohol spectrum disorder (FASD). FASD is comprised of four categorical entities summarized in Table 1 [6]. Current prevalence estimates suggest that $1 \%$ of live births may have an FASD and the rate is much higher in some at-risk populations [8].

While prenatal alcohol exposure may result in damage to any of the organ systems, the heart and brain appear to be two most commonly reported organ systems [6] [9]-[13]. However, recently Patel et al. [14] have reported that they found no increase in risk for non-syndromic atrioventricular septal defects attributable to maternal alcohol consumption in a large data set from the National Birth defects Prevention Study.

While multiple studies have examined the role of prenatal alcohol exposure on fetal and infant mortality, very limited data are available on mortality in FASD. To improve understanding of mortality events in FASD, we completed a review of published papers reporting on deaths in people with an FASD.

\section{Methods}

A search strategy was developed and conducted by an expert reference librarian with guidance from the authors. The search was conducted through the following electronic databases and search engines: PubMed, Scopus, Cochrane Database of Systematic Reviews, Quertle, and Google Scholar (Table 2). The following key words and subject headings were utilized in the search: prenatal alcohol exposure, fetal alcohol syndrome, mortality and prenatal exposure delayed effects. The reference list for each paper was then hand searched to identify other potential references not identified by our electronic search strategy. We excluded studies that did not report data on FASD.

The search, which was completed to December of 2012, placed no limits on language or publication date. When examining data from the selected articles, we relied on the original authors' report for prenatal alcohol exposure, cause of death and case descriptions (birth defects, infectious illness).

\section{Results}

We located 26 papers which met our inclusion criteria and reported on 57 deaths (Table 2). The reports ranged from single cases to 13 deaths. Table 2 also summarizes data on prenatal exposure, gestational age at birth, birth weight, growth impairment, abnormal facial features, neurocognitive impairments and FASD ca-

Table 1. Diagnostic criteria for Fetal Alcohol Spectrum Disorders (FASD) ${ }^{1}$.

\begin{tabular}{|c|c|c|c|c|}
\hline & $\begin{array}{c}\text { CNS } \\
\text { Microcephaly Malformations } \\
\text { Cognitive Impairments Mental } \\
\text { Disorders Developmental Delays }\end{array}$ & $\begin{array}{l}\text { FACE } \\
\text { Thin upper lip Philtrum abnormalities } \\
\text { Short palpebral fissures Flat nasal bridge } \\
\text { Epicanthal folds Cleft lip and/or palate }\end{array}$ & $\begin{array}{c}\text { GROWTH } \\
\text { Height }<10 \text { th } \\
\text { Weight }<10 \text { th } \\
\text { Head Circumference }<10 \text { th }\end{array}$ & EXPOSURE \\
\hline FAS & YES (3 + Domain Deficits) & YES (3 of 3) & YES $(\leq 10 \%)$ & $\mathrm{YES}^{2}$ \\
\hline PFAS & YES (3 + Domains Deficits) & YES (2 of 3) & N/A & $\mathrm{YES}^{2}$ \\
\hline ARND & YES (2 + Domain Deficits) & N/A & N/A & YES \\
\hline ARBD $^{3}$ & & & & YES \\
\hline
\end{tabular}

${ }^{1}$ FAS = Fetal alcohol syndrome; PFAS = Partial fetal alcohol syndrome; ARND = Alcohol related neurodevelopmental disorders; ARBD = Alcohol related birth defects. ${ }^{2}$ Diagnosis can be made without confirmation of prenatal alcohol exposure. ${ }^{3}$ Birth defects attributed to prenatal alcohol exposure. Defects of the heart and brain are the most prevalent. 
Table 2. The search strategies utilized in this review at each sequential step - duplicates were excluded at each subsequent step.

\begin{tabular}{|c|c|}
\hline SEARCH STRATEGY \& TERM & YIELD (N) \\
\hline \multicolumn{2}{|l|}{ Pub Med Searches } \\
\hline "Fetal Alcohol Syndrome/mortality”[Mesh] & 2 \\
\hline (“Fetal Alcohol Syndrome”[Mesh]) AND “Infant Mortality”[Mesh] & 3 \\
\hline (“Fetal Alcohol Syndrome”[Mesh]) AND “Sudden Infant Death”[Mesh] & 3 \\
\hline “Stillbirth”[Mesh] AND “Ethanol”[Mesh] & 0 \\
\hline (“Sudden Infant Death”[Mesh Terms]) AND “Ethanol”[MeSH Terms] & 0 \\
\hline “Survival Analysis” [mesh] AND “Fetal Alcohol Syndrome” [mesh] & 1 \\
\hline (“Cause of Death”[MeSH Terms]) AND “Fetal Alcohol Syndrome”[Mesh] & 1 \\
\hline (“Fetal Alcohol Syndrome”[Mesh] OR “Alcoholism”[Mesh]) AND “Cause of Death”[Mesh] & 2 \\
\hline (“Fetal Alcohol Syndrome”[Mesh]) AND “Fetal Death”[Mesh] & 6 \\
\hline (“Alcoholism/complications”[MeSH Terms]) AND “Fetal Death/chemically induced”[MeSH Terms] & 5 \\
\hline $\begin{array}{l}\text { (((“Fetal Diseases/chemically induced”[MeSH Terms]) AND (alcohol OR ethanol[Title/Abstract]))) AND } \\
\text { (autopsy OR death OR fatal* OR mortal*) }\end{array}$ & 7 \\
\hline (“Fetal Alcohol Syndrome”[Mesh]) AND autopsy[Title/Abstract] & 3 \\
\hline ("Fatal Outcome”[Mesh]) AND “Fetal Alcohol Syndrome”[Mesh] & 3 \\
\hline (((“Death”[Mesh]) And “Fetal Alcohol Syndrome”[Mesh]))) & 5 \\
\hline \multicolumn{2}{|l|}{ Fetal alcohol spectrum disorders mortality } \\
\hline "fetal alcohol” AND death & 4 \\
\hline (“fetal alcohol”[Title/Abstract]) AND (death OR mortality[Title/Abstract]) & 2 \\
\hline (fetal alcohol effects) AND death & 1 \\
\hline Foetel alcohol death & 5 \\
\hline Embryofetal alcohol syndrome & 1 \\
\hline Hand Search of Bibliographies & 12 \\
\hline Scopus & 4 \\
\hline Cumulative Index to Nursing and Allied Health Literature (CINAHL) & 2 \\
\hline Academic Search Premier & 53 \\
\hline
\end{tabular}

tegorical diagnosis. The diagnostic criteria for the FASD include growth impairment, abnormal facial features and neurocognitive impairments (Table 3).

Data on prenatal alcohol exposure was present for 48 of the 57 cases (84.2\%) and all of these women were reported to be heavy drinkers. Drinking was most prevalent in the first trimester. The 57 cases included data on birth weight for 20 cases (35.1\%) and gestational age at delivery for 22 cases (38.6\%). Of the cases with information on gestational age, 12 of $22(63.6 \%)$ were born preterm ( $<37$ weeks gestation). Data was available on weight for 11 cases (19.3\%), on height for $6(10.5 \%)$ and on head circumference for 12 cases (21.1\%). Nonspecific growth restriction was reported in 9 cases (15.8\%).

The three most frequently reported abnormal facial features in fetal alcohol syndrome are thin upper lip, indistinct or smooth philtrum and short palpebral fissure length (Table 3). Abnormal facial features were reported for 32 (56.1\%) of the cases. These were highly variable but included short palpebral fissures 13 (22.8\%), thin upper lip 9 (15.8\%), microganthia 8 (14\%), low set ears 7 (12.3\%), ptosis 7 (12.3\%), absent or indistinct philtral ridge 5 (8.8\%), epicanthal folds 5 (8.8\%), cleft palate 9 (15.8\%), flat nasal bridge 6 (10.5\%) and midface hypoplasia 3 (5.3\%) (Table 2). In addition 10 cases (17.5\%) were reported to have unspecified abnormal facial features, including FAS typical facial abnormalities, diagnostic facial features or dysmorphic facial features.

The neurocognitive features commonly reported in FASD are microcephaly, intellectual disability, attention deficit hyperactivity disorder and behavioral impairments. Structural abnormalities of the central nervous system were reported for 25 of the 49 cases (51\%). These included microcephaly, agenesis/absence of the corpus callo- 
Table 3. Data on exposure, gestational age at birth, birth weight, growth impairments, and abnormal facial features for 57 deaths in people with a fetal alcohol spectrum disorder $(\mathrm{HT}=$ height, $\mathrm{HC}=$ head circumference, $\mathrm{WT}=$ weight, $\mathrm{FP}=$ flat philtrum, EP = epicanthal folds, SPL = short palpebral fissures length, CC = chest circumference, TUL = thin upper lip).

\begin{tabular}{|c|c|c|c|c|c|c|}
\hline Publication & $\begin{array}{l}\text { Alcohol } \\
\text { Exposure } \\
+=\text { reported }\end{array}$ & $\begin{array}{l}\text { Gestational } \\
\text { Age/Birth } \\
\text { Weight }\end{array}$ & $\begin{array}{l}\text { Growth } \\
\text { Impairment }\end{array}$ & Facial Features & Neurocognitive Impairments & $\begin{array}{l}\text { FASD } \\
\text { Status }\end{array}$ \\
\hline$[34]$ & + & $\begin{array}{l}\text { Term } \\
1600 \mathrm{~g}\end{array}$ & $\begin{array}{l}\text { WT, } \mathrm{HC}<10 \% \text { at } \\
\text { birth }\end{array}$ & $\begin{array}{l}\text { SPL, FP, TUL, micrognathia } \\
\text { and low set ears }\end{array}$ & $\begin{array}{l}\text { Brain was histologically normal on } \\
\text { autopsy }\end{array}$ & FAS \\
\hline$[35]$ & + & $\begin{array}{l}35 \text { weeks } \\
1360 \mathrm{~g}\end{array}$ & $\begin{array}{l}\text { HC, HT, WT }<3 \% \\
\text { at birth }\end{array}$ & $\begin{array}{l}\text { Hypertelorism, broad nasal } \\
\text { bridge, inferolateral deviation of } \\
\text { right eye, low-set and } \\
\text { posteriorly rotated right ear, }\end{array}$ & $\begin{array}{l}\text { Microcephaly and partial agenesis of } \\
\text { the corpus callosum }\end{array}$ & FAS \\
\hline$[36]$ & + & & Reported & Described as abnormal & & FAS \\
\hline$[36]$ & + & & Reported & Described as abnormal & & FAS \\
\hline [37] & + & $\begin{array}{l}37 \text { weeks } \\
2330 \mathrm{~g}\end{array}$ & $\begin{array}{l}\text { WT, HT, HC, CC } \\
<\text { rd \% }\end{array}$ & $\begin{array}{l}\text { Long upper lip, cleft palate, } \\
\text { hypertelorism, partial ptosis, } \\
\text { curved fused eyebrows }^{1}\end{array}$ & $\begin{array}{l}\text { Absence of corpus callosum and } \\
\text { underdevelopment of cerebellar } \\
\text { vermis, areas of necrosis in the brain }{ }^{2}\end{array}$ & FAS \\
\hline [37] & + & $\begin{array}{l}32 \text { weeks } \\
1180 \mathrm{~g}\end{array}$ & $\begin{array}{l}\text { WT, HT, HC, and } \\
\text { CC }<3 \text { rd \% }\end{array}$ & $\begin{array}{l}\text { Reportedly similar to previous } \\
\text { case }\end{array}$ & $\begin{array}{l}\text { Brain was small for developmental age } \\
\text { (395 grams at } 4 \text { months) }^{3}\end{array}$ & FAS \\
\hline$[37]$ & + & $\begin{array}{l}\text { Term } \\
\text { Not reported }\end{array}$ & & $\begin{array}{l}\text { Similar to previous cases } \\
\text { without cleft palate }\end{array}$ & $\begin{array}{l}\text { Abnormalities of cortical mantle } \\
\text { formation }^{4}\end{array}$ & FAS \\
\hline [37] & + & $\begin{array}{l}35 \text { weeks } \\
1525 \mathrm{~g}\end{array}$ & $\begin{array}{l}\text { WT }(1525 \mathrm{~g}), \mathrm{HC} \\
\text { (28.5) both small } \\
\text { for gestational age }\end{array}$ & $\begin{array}{l}\text { Nondiagnostic facial features } \\
\text { (flat nasal bridge and recessive } \\
\text { mandible) }\end{array}$ & Brain was heavy (405g) but small ${ }^{5}$ & FAS \\
\hline [37] & + & $\begin{array}{l}29 \text { weeks } \\
880 \mathrm{~g}\end{array}$ & WT below 3rd \% & $\begin{array}{l}\text { Reported facial features to be } \\
\text { similar to the other cases in the } \\
\text { paper }\end{array}$ & $\begin{array}{l}\text { Brain small for gestational age ( } 124.5 \\
\text { g) and an area of heterotopia white } \\
\text { matter of the temporal lobe }\end{array}$ & FAS \\
\hline [38] & + & $\begin{array}{l}\text { Term } \\
1770 \mathrm{~g}\end{array}$ & $\begin{array}{l}\text { Reduced birth } \\
\text { weight, growth } \\
\text { deficiency at } 2 \\
\text { years }\end{array}$ & $\begin{array}{l}\text { Blepharophimosis, SPL, flat } \\
\text { nasal bridge, bilateral ptosis, } \\
\text { and EF }\end{array}$ & $\begin{array}{l}\text { Microdysplasias (partial fusion of third } \\
\text { ventricle), spongiform loosening in the } \\
\text { optic nerve and in the hypothalamus, } \\
\text { seizures, psychomotor retardation }\end{array}$ & FAS \\
\hline$[38]$ & + & Term & & $\begin{array}{l}\text { Blepharophimosis, } \\
\text { hypertelorism, micrognathia, } \\
\text { hypoplastic ears, short upturned } \\
\text { nose, and cleft palate }\end{array}$ & $\begin{array}{l}\text { Hydrocephalus internus, heterotopic } \\
\text { gyri in cerebellar white matter, slowed } \\
\text { maturation of cerebral and cerebellar } \\
\text { white matter }\end{array}$ & FAS \\
\hline [38] & + & & & SPL, TUL, and low set ears & $\begin{array}{l}\text { Seizures, porencephaly, hydrocephaly, } \\
\text { agenesis of corpus callosum, multiple } \\
\text { other abnormalities }\end{array}$ & FAS \\
\hline$[26]$ & + & & & & & FAS \\
\hline$[26]$ & + & & & & & FAS \\
\hline$[26]$ & + & & & & & FAS \\
\hline$[26]$ & + & & & & & FAS \\
\hline$[26]$ & + & & & & & FAS \\
\hline [26] & + & & & & & FAS \\
\hline [26] & + & & & & & FAS \\
\hline [26] & + & & & & & FAS \\
\hline [26] & + & & & & & FAS \\
\hline [26] & + & & & & $\begin{array}{l}\text { Status epilepticus at } 18 \text { months, } \\
\text { hemiplegia }\end{array}$ & FAS \\
\hline [26] & + & & & & & FAS \\
\hline [26] & + & & & & & FAS \\
\hline
\end{tabular}




\section{Continued}

\begin{tabular}{|c|c|c|c|c|c|c|}
\hline [26] & + & & & & & FAS \\
\hline [39] & + & $\begin{array}{l}36 \text { to } 37 \\
\text { weeks } \\
3100 \mathrm{~g}\end{array}$ & $\begin{array}{l}\text { At death growth } \\
\text { was } 10 \text { th } \% \text { and } \\
\text { HC was } 5 \text { th \% }\end{array}$ & $\begin{array}{l}\text { SPL, hypertelorism, midfacial } \\
\text { hypoplasia, high arched palate }\end{array}$ & $\begin{array}{l}\text { Microencephaly, small anteriorly fused } \\
\text { frontal lobes }^{6}\end{array}$ & FAS \\
\hline [31] & + & $\begin{array}{l}32 \text { weeks } \\
1300 \mathrm{~g}\end{array}$ & None & Microphthalmia, cleft palate & Agenesis of corpus callosum ${ }^{7}$ & FAS \\
\hline [28] & + & & & & & FAS \\
\hline [40] & + & 32 weeks & $\begin{array}{l}\text { prenatal growth } \\
\text { deficiency }\end{array}$ & SPL, maxillary hypoplasia & Microcephaly ${ }^{8}$ & FAS \\
\hline [40] & + & $\begin{array}{l}\text { Term } \\
3200 \mathrm{~g}\end{array}$ & None & $\begin{array}{l}\text { SPL, maxillary hypoplasia, EF, } \\
\text { and micrognathia }\end{array}$ & $\begin{array}{l}\text { Hydrocephalus at birth, no cranial } \\
\text { nerve function, hypotonia, areflexia }\end{array}$ & $\begin{array}{l}\text { FASD/ } \\
\text { ARND }\end{array}$ \\
\hline [41] & + & & & “Typical facial anomalies” & $\begin{array}{l}\text { Mild hydrocephalus internus, multiple } \\
\text { heterotopic gyri of white matter of } \\
\text { cerebellum }\end{array}$ & FAS \\
\hline [41] & + & & & “Typical facial anomalies” & $\begin{array}{l}\text { Severely mentally disabled, partial } \\
\text { fusion of the 3rd ventricle }{ }^{10}\end{array}$ & FAS \\
\hline [41] & + & & & "Typical facial anomalies” & $\begin{array}{l}\text { Extreme brain malformations, absent } \\
\text { corpus callosum }^{11}\end{array}$ & FAS \\
\hline [42] & + & $\begin{array}{l}34 \text { weeks } \\
1640 \mathrm{~g}\end{array}$ & $\begin{array}{l}\text { WT and HT at } \\
10 \text { th } \% \text { for } 34 \\
\text { weeks, HC } 10 \% \text { - } \\
\text { 25th \% }\end{array}$ & $\begin{array}{l}\text { SPL, microphthalmia, low nasal } \\
\text { bridge with upturned nose, } \\
\text { narrow face with triangular chin }\end{array}$ & None reported & FAS \\
\hline [43] & + & $3600 \mathrm{~g}$ & None & $\begin{array}{l}\text { SPL, TUL, hypoplastic philtrum, } \\
\text { short anteverted nose, and } \\
\text { micrognathia }\end{array}$ & Mental retardation & FAS \\
\hline [44] & + & 40 weeks & $\begin{array}{l}\text { prenatal growth } \\
\text { deficiency }\end{array}$ & $\begin{array}{l}\text { SPL, EF, small lips, } \\
\text { micrognathia, small mandible, } \\
\text { posteriorly rotated ears }\end{array}$ & $\begin{array}{l}\text { Microcephaly, occasional glial } \\
\text { meningeal heterotopias, cortical } \\
\text { cerebellar heterotopias }^{12}\end{array}$ & FAS \\
\hline [45] & + & $\begin{array}{l}34 \text { weeks } \\
1780 \mathrm{~g}\end{array}$ & $\begin{array}{l}\text { WT and HT } \\
\text { 10th \%, HC }<3 \% \\
\text { at birth }\end{array}$ & $\begin{array}{l}\text { SPL, long, FP, micrognathia, } \\
\text { low-set ears, short upturned } \\
\text { nose, flat nasal bridge, and } \\
\text { high-arched palate }\end{array}$ & $\begin{array}{l}\text { Opisthotonic posturing, increased tone, } \\
\text { brisk deep tendon reflexes, absent } \\
\text { social smile at } 7 \text { weeks }\end{array}$ & FAS \\
\hline [46] & & & & & $\begin{array}{l}\text { Microencephaly, agenesis of corpus } \\
\text { callosum }^{13}\end{array}$ & FAS \\
\hline [46] & & & & & $\begin{array}{l}\text { Microencephaly, severe glioneuronal } \\
\text { heterotopias in over normal cortex }\end{array}$ & FAS \\
\hline [47] & + & $\begin{array}{l}35 \text { weeks } \\
1450 \mathrm{~g}\end{array}$ & $\begin{array}{l}\text { At birth WT, HT, } \\
\text { HC }<10 \text { th } \%\end{array}$ & $\begin{array}{l}\text { SPL, FP, TUL, broad nose, low } \\
\text { set ears, midface hypoplasia, } \\
\text { hypertelorism }\end{array}$ & $\begin{array}{l}\text { Pachygyria, triphyllocephaly semilobar } \\
\text { holoprosencephaly }\end{array}$ & FAS \\
\hline [48] & + & $\begin{array}{l}40 \text { weeks } \\
1970 \mathrm{~g}\end{array}$ & Present & Present & Present & FAS \\
\hline [48] & + & $\begin{array}{l}32 \text { weeks } \\
2110 \mathrm{~g}\end{array}$ & Present & Present & Present & FAS \\
\hline [48] & + & $\begin{array}{l}36 \text { weeks } \\
2420 \mathrm{~g}\end{array}$ & Present & Present & Present & FAS \\
\hline [49] & + & $\begin{array}{l}36 \text { weeks } \\
1500 \mathrm{~g}\end{array}$ & $\begin{array}{l}\text { Severe growth } \\
\text { impairment }\end{array}$ & $\begin{array}{l}\text { Moderately pronounced cranial } \\
\text { dysmorphism }\end{array}$ & $\begin{array}{l}4 \text { mamillary bodies } \\
\text { At } 4 \text { months brain weight } 472 \mathrm{~g}\end{array}$ & FAS \\
\hline [50] & + & $1440 \mathrm{~g}$ & & & Brain hemorrhage & FAS \\
\hline [51] & + & & None reported & Dysmorphic facies & Psychomotor retardation & FAS \\
\hline [52] & + & 28 weeks & & $\begin{array}{l}\text { Flat nose, cleft lip and palate, } \\
\text { hypertelorism }\end{array}$ & $\begin{array}{l}\text { Microcephaly, Hypotonia, recurrent } \\
\text { myoclonic seizures, alobar } \\
\text { holoprosencephaly }^{14}\end{array}$ & $\begin{array}{c}\text { Possible } \\
\text { FAS }\end{array}$ \\
\hline [53] & + & & $\begin{aligned} \text { WT } & <10 \text { th } \%, \text { HC } \\
& <50 \text { th } \%\end{aligned}$ & FP, SPL, ptosis & Mental retardation & FAS \\
\hline [54] & + & $1446 \mathrm{~g}$ & & $\begin{array}{l}\text { EF, hypertelorism, facial } \\
\text { asymmetry, esotropia }\end{array}$ & $\begin{array}{l}\text { Occipital cavernous hemangioma } \\
\text { delayed/speech and development }\end{array}$ & FAS \\
\hline [22] & & & & & & FAS \\
\hline
\end{tabular}




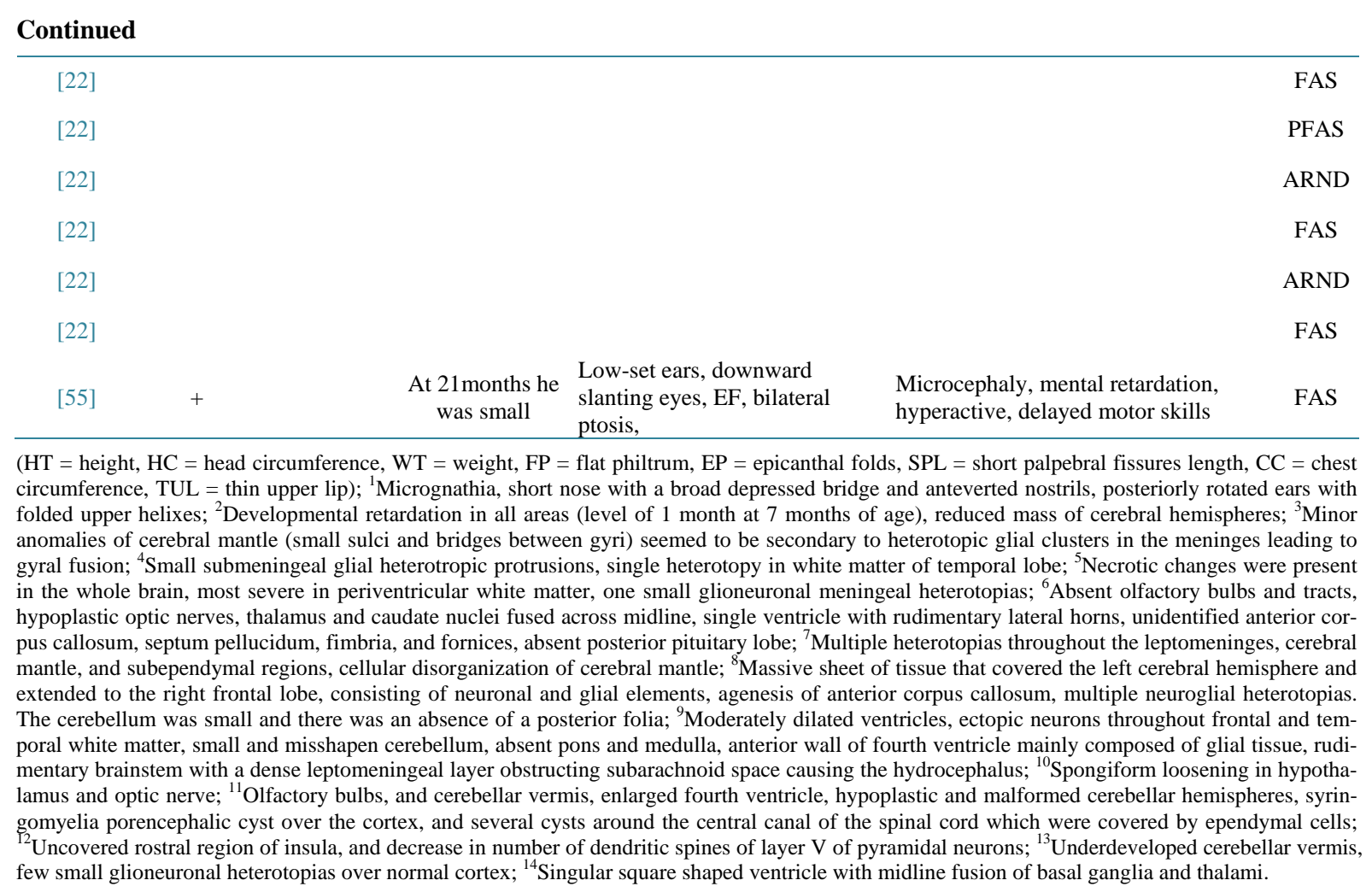

sum and multiple severe brain malformations described in Table 2.

In Table 4 we summarize the data on age at death, gender, and cause of death. Figure 1 is a graphic presentation of the age at death data demonstrating that $12.7 \%$ of deaths occurred in the first week, and $54.5 \%$ of the deaths occurred by age one year. Only $25.5 \%$ of the deaths were reported to occur after 5 years of age (Figure 1). Gender was reported for 50 cases; 29 (58\%) were male and 21 (42\%) were female. The M/F ratio was 1.38 males for each female.

In Table 5 we summarize the leading causes of death which was available for 49 of the 57 cases (86\%). The most common cause of death was congenital heart disease $37 / 49$ (75.5\%) with suspected or confirmed congenital heart disease including atrial septal defect, patent ductus arteriosus, tetralogy of Fallot, hypoplastic left heart, aortic arch interruption, etc. (Table 4). However, for some cases it was difficult to determine if the heart defect was the cause of death, a complicating condition, or related postmortem finding.

The second leading cause of death was congenital brain malformations which were present in 25/49 (51\%). These included microcephaly, abnormalities of the corpus callosum, hydrocephalus, porencephaly and multiple other less severe malformations. Again it was difficult to determine if these defects were the cause of death, a complicating condition, or a postmortem finding of uncertain significance. Sepsis 7/49 cases (14.3\%), malformations of the kidney $7 / 49$ cases (14.3\%), and cancer $4 / 49$ cases (8.2\%) were the other leading causes of death.

\section{Discussion}

We identified 57 deaths occurring in people with an FASD and identified a potential cause of death for 49 (86\%). These 26 studies reported highly variable dosimetry for prenatal alcohol exposure, thus no dose response data were available to estimate mortality risk by duration of exposure, pattern of drinking, or quantity of exposure.

In this review congenital heart defects were reported to be the most prevalent cause of mortality in people with an FASD. We have previously reviewed the published data on the association of FASD and congenital heart defects [15]. In several cases it was difficult to determine if the deaths were due to congenital heart defects which were morbid but not mortal. However, several severe and likely lethal cardiac defects were reported including tetralogy of Fallot, hypoplastic left heart and aortic arch interruption. Another potential reason for the 
Table 4. Data on age at death, gender and cause of death for 57 deaths in people with fetal alcohol spectrum disorders.

\begin{tabular}{|c|c|c|c|c|}
\hline Publication & Age of Death & Gender & Cause of Death & Comments \\
\hline [34] & 20 hours & Female & & $\begin{array}{l}\text { Baby was born with phocomelia of upper limbs } \\
\text { and amelia of lower limbs }\end{array}$ \\
\hline [35] & 110 days & Male & Aspiration & Autopsy found a paravertebral neuroblastoma. ${ }^{1}$ \\
\hline$[36]$ & & & $\begin{array}{l}\text { Heart failure due to combined atrial and ventricular } \\
\text { septal defects and persistent ductus arteriosus }\end{array}$ & \\
\hline [36] & & & Bowel obstruction from an inguinal hernia & $\begin{array}{l}\text { Large ventricular septal defect and a persistent } \\
\text { left superior vena cava. }\end{array}$ \\
\hline [37] & 8 months & Female & $\begin{array}{l}\text { Congestive heart failure (ventricular septal defect) } \\
\text { with superimposed infection (Yersinia) }\end{array}$ & $\begin{array}{l}\text { Multiple abnormalities: hypoplastic nipples, } \\
\text { hypoplasia of the labia majora, clitoral } \\
\text { hypertrophy, deep sacral dimple }\end{array}$ \\
\hline [37] & 4 months & Male & $\begin{array}{l}\text { Autopsy - ventricular septal defect, cardiomegaly, } \\
\text { and collapse of left lung }\end{array}$ & \\
\hline [37] & 48 hours & Male & Autopsy - atrial septal defect and cardiomegaly & \\
\hline [37] & 17 days & Male & Cardiac arrest, acidosis & \\
\hline [37] & 4 days & Male & $\begin{array}{l}\text { Autopsy - diffuse pulmonary hemorrhage and } \\
\text { overdistention of lungs with cyst formation }\end{array}$ & \\
\hline$[38]$ & 4.5 years & Male & $\begin{array}{l}\text { Complete atrioventricular channel with infundibular } \\
\text { and valvular stenosis of the pulmonary artery and } \\
\text { bicuspid pulmonic valve }\end{array}$ & Clinodactyly of 5th finger \\
\hline [38] & 6 months & Male & $\begin{array}{l}\text { Autopsy found Tetralogy of Fallot, thrombosis of } \\
\text { basilar artery, and necrosis of cerebellum and pons }\end{array}$ & Aplasia of right kidney, hydronephrosis ${ }^{2}$ \\
\hline [38] & 9 months & Female & $\begin{array}{l}\text { Autopsy showed atrial septal defect, aspiration } \\
\text { pneumonia, and multifocal interstitial pneumonia }\end{array}$ & Hydrocephalus, severe hypotonia ${ }^{3}$ \\
\hline$[26]$ & $\begin{array}{l}5 \text { years, } \\
5 \text { months }\end{array}$ & Female & Tetralogy of Fallot & \\
\hline$[26]$ & $\begin{array}{l}4 \text { years, } \\
4 \text { months }\end{array}$ & Male & Tetralogy of Fallot & \\
\hline$[26]$ & $\begin{array}{l}5 \text { years, } \\
3 \text { months }\end{array}$ & Male & Tetralogy of Fallot & \\
\hline$[26]$ & $\begin{array}{c}1 \text { year, } \\
3 \text { months }\end{array}$ & Male & H. influenzae meningitis & \\
\hline$[26]$ & $\begin{array}{l}5 \text { years } \\
9 \text { months }\end{array}$ & Male & Tetralogy of Fallot & \\
\hline$[26]$ & $\begin{array}{l}1 \text { year, } \\
11 \text { months }\end{array}$ & Male & Bronchopneumonia, septicemia & \\
\hline$[26]$ & $\begin{array}{l}10 \text { years, } \\
7 \text { months }\end{array}$ & Male & Tetralogy of Fallot & Cleft palate \\
\hline$[26]$ & 5 months & Male & Congenital extra hepatic biliary atresia & $\begin{array}{l}\text { Ventricular and atrial septal defects, patent } \\
\text { ductus arteriosus }\end{array}$ \\
\hline$[26]$ & 13 days & Female & Hypoplastic left heart syndrome & \\
\hline$[26]$ & $\begin{array}{l}29 \text { years, } \\
10 \text { months }\end{array}$ & Female & Congenital cirrhosis & $\begin{array}{l}\text { Esophageal varices, portocaval shunts, } \\
\text { splenectomy }\end{array}$ \\
\hline$[26]$ & 26 months & Female & Tracheolaryngomalacia, laryngeal webbing & Gastroesophageal reflux, pulmonary aspiration \\
\hline [39] & 2.5 months & Female & Respiratory distress & Choanal stenosis $^{4}$ \\
\hline [31] & 5 days & Female & Multiple apneic episodes & $\begin{array}{l}2 \text { vessel umbilical cord, left sternal border } \\
\text { murmur }^{5}\end{array}$ \\
\hline [28] & 3.5 years & Female & Drowning in bath tub & $\begin{array}{l}4 / 6 \text { systolic murmur interpreted as a ventricular } \\
\text { septal defect }{ }^{6}\end{array}$ \\
\hline$[40]$ & 6 weeks & Female & Prolonged apneic spell & Joint and cardiac anomalies \\
\hline$[40]$ & 10 weeks & Male & Cardiopulmonary arrest & \\
\hline$[40]$ & 68 hours & & Idiopathic respiratory distress syndrome & \\
\hline$[41]$ & $\begin{array}{c}\text { Either } \\
6 \text { months or } \\
4 \text { years } \\
\text { Either }\end{array}$ & Male & & Tetralogy of Fallot, hypoplasia of one kidney \\
\hline$[41]$ & $\begin{array}{l}6 \text { months or } \\
4 \text { years }\end{array}$ & Male & Heart failure & Atrioventricular canal \\
\hline [41] & 9 months & & Massive hydrocephalus internus and externus & Atrial septal defect \\
\hline
\end{tabular}




\section{Continued}

\begin{tabular}{|c|c|c|c|c|}
\hline [42] & 7 days & Male & Shock & $\begin{array}{l}\text { Interrupted aortic arch with aortopulmonary } \\
\text { fenestration }\end{array}$ \\
\hline [43] & 11 months & Male & Pulmonary Hemorrhage & $\begin{array}{l}\text { Severe mitral regurgitation, mild-moderate aortic } \\
\text { stenosis and pulmonary stenosis }{ }^{7}\end{array}$ \\
\hline [44] & 4 months & Male & Pneumonia and congestive heart failure & $\begin{array}{l}2 \mathrm{~cm} \text { ventricular septal defect with ventricular } \\
\text { hypertrophy }\end{array}$ \\
\hline [45] & 96 days & Male & Sepsis & $\begin{array}{l}\text { Atrial septal defect, pyloric stenosis with failure } \\
\text { to thrive, undescended testes, sacral dimple }\end{array}$ \\
\hline [46] & 8 months & & Congenital heart disease causing heart failure & Hydronephrosis \\
\hline [46] & 4 months & & Congenital heart disease causing heart failure & Hydronephrosis \\
\hline [47] & 41 days & Female & Cardiopulmonary failure & Pfeiffer-like syndrome ${ }^{8}$ \\
\hline$[48]$ & 5 months & Female & Reported as noncardiac & Atrial septal defect \\
\hline [48] & 9 months & Female & Reported as noncardiac & Atrial septal defect \\
\hline$[48]$ & 4 months & Male & Hypoxic spells and respiratory failure & Pulmonary stenosis, ventricular septal defect \\
\hline [49] & 4 months & Male & Unknown, found dead in crib & $\begin{array}{l}\text { Ventricular septal defect, radial and ulnar } \\
\text { malformation hospitalized for first } 3 \text { months life }\end{array}$ \\
\hline$[50]$ & 10 hours & Male & Brain hemorrhage & $\begin{array}{l}\text { Pulmonary hyaline membranes and a left } \\
\text { undescended testicle }\end{array}$ \\
\hline$[51]$ & 27 months & Male & Candida Sepsis & $\begin{array}{l}\text { Born with dysplastic kidney and required renal } \\
\text { transplant and immunosupressive therapy, } \\
\text { developed hepatoblastoma }\end{array}$ \\
\hline$[52]$ & 8 days & & Recurrent Seizures & Patent ductus arteriosus \\
\hline [53] & 21 months & Male & Septicemia & Embryonal rhabdoymasarcoma \\
\hline$[54]$ & 13 years & Female & Adrenal carcinoma & \\
\hline$[22]$ & 17 years & Female & Accident & \\
\hline$[22]$ & 22 years & Female & Complications from cerebral palsy & \\
\hline$[22]$ & 11 years & Female & Respiratory complications & \\
\hline$[22]$ & 17 years & Female & Suicide & \\
\hline$[22]$ & 28 years & Male & Congenital heart disease & \\
\hline$[22]$ & 23 years & Female & & \\
\hline$[22]$ & 18 years & Male & & \\
\hline [55] & 21 months & Male & $\begin{array}{l}\text { Septic complications from rhabdomyosarcoma } \\
\text { of the bladder }\end{array}$ & Kidney-ureter abnormalities, urinary infection ${ }^{9}$ \\
\hline
\end{tabular}

${ }^{1}$ Multiple other defects including multiple atrial septal defects, incomplete rotation of the gut with failure of fixation of the cecum and descending colon, right diaphragmatic hernia and bilateral inguinal hernias, as well as midline cleft palate, cleft lip on the left, wide spaced nipples, prominent right hemithorax, simian creases, hypoplastic nails, long and slender fingers, syndactyly of the second and third toes bilaterally, undescended testes, synostosis of the right radius and ulna; ${ }^{2}$ Radial ulnar synostosis, camptodactyly, and anomalies of the palmar creases; ${ }^{3}$ Porencephaly diastasis recti, hypertrophy of clitoris, anomalies of palmar creases. Hydramnios, cephalohematoma, and generalized edema noted at birth; ${ }^{4}$ Transverse palmar crease, bilateral renal parenchymal duplication with single pelvis, small atrial septal defect, severe thymic and adrenal involution; ${ }^{5} \mathrm{Hirsutism} \mathrm{(especially} \mathrm{over}$ forehead), overlapping of 2nd by the 3rd fingers, clinodactyly of the left 5th finger, camptodactyly of right 3rd finger, absence of distal interphalangeal crease; ${ }^{6}$ Congenital hip dysplasia, repeated ear infections; ${ }^{7}$ Short 5 th fingers, palmar transverse creases, congenital heart defect - markedly thickened and rolled up mitral valve, moderately thickened aortic valve leaflets, dysplastic pulmonary valve, markedly dilated left atrium, severe hypertrophy of left ventricle, moderate hypertrophy of right ventricle, patent foramen ovale; ${ }^{8}$ Cloverleaf skull, craniosynostosis, bilateral choanal stenosis, bilateral auditory canal bony atresia, hypoplasia of stapes, cleft palate, short neck, cubitus valgus, broad thumbs, bilateral palmar creases, broad and medially deviated halluces, sacral and coccygeal deformities, caudal regression, patent ductus arteriosus, left-sided aortic arch and aberrant retroesophageal right subclavian artery; ${ }^{9}$ Broad nose, anteverted nares, marked nasiolabial folds, high palate.

disproportionate numbers of cardiac deaths may be due to the publication of multiple studies specifically focused on cardiac malformations in FASD, primarily fetal alcohol syndrome.

The number and severity of brain malformations was also interesting. These include hydrocephalus, porencephaly, absence of the corpus callosum, semilobar holoprosencephaly, and brain hemorrhage (Table 2). These findings suggest that pediatric neurologists and neurologists are likely to encounter and manage cases of FASD with some frequency [16] [17].

Death from sepsis was reported for 7/49 cases (14.3\%). Susceptibility to infection and increased risk of sepsis 


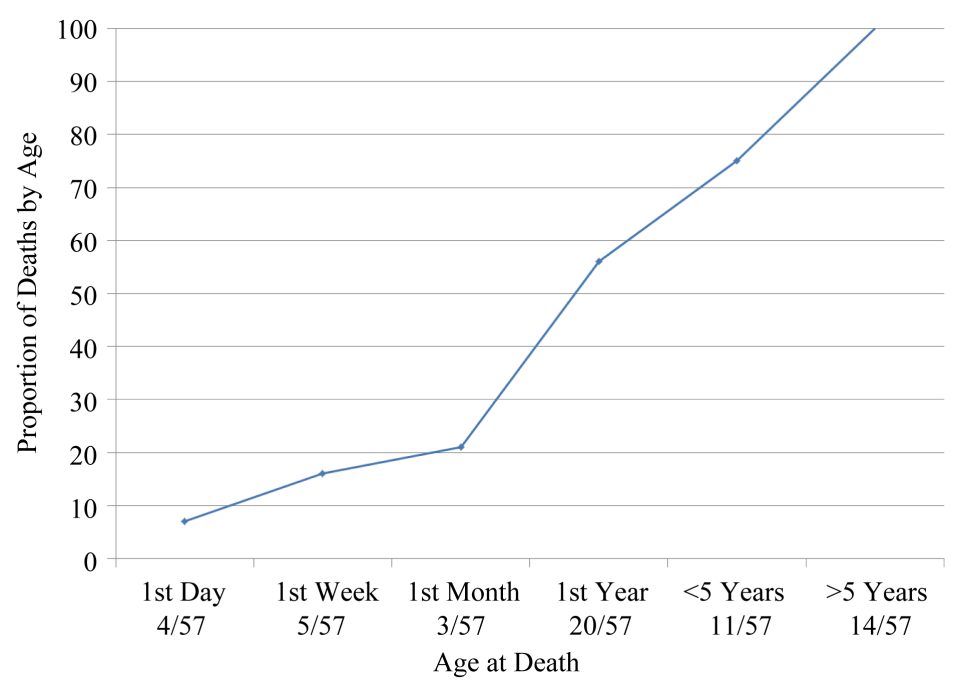

Figure 1. Cumulative mortality by age.

Table 5. Cause of death findings for 45 of the 59 cases included in this review.

\begin{tabular}{ccc}
\hline & $\mathrm{n}$ & $\%$ \\
\hline Congenital Heart Malformations & $37 / 49$ & 75.5 \\
Congenital Brain Malformations & $25 / 49$ & 51 \\
Sepsis & $7 / 49$ & 14.3 \\
Kidney Malformations & $7 / 49$ & 14.3 \\
Cancer & $4 / 49$ & 8.2 \\
Liver Malformations & $2 / 49$ & 4.1 \\
\hline
\end{tabular}

in FASD has been previously reported [10] [18]-[22]. Kidney malformation was found to be the cause of death in $7 / 49$ cases (14.3\%). Previous studies have examined malformations of the kidney, however, it is difficult to determine if the reported cases exceed expected rates for the co-occurrence of two conditions or if rates of kidney malformations exceed expected rates in people with an FASD [9] [23] [24]. Cancers were reported to be a cause of death in $4 / 49$ cases (8.2\%). However, no particular risk for a specific cancer could be determined from this small sample.

The early age of death distribution was surprising since a diagnosis of FASD is difficult in infancy and early childhood. Given the prevalence of FASD at about 1\% of live births case reports and case series studies of mortality should be common rather than limited to 57 cases. It seems likely that most cases of mortality in FASD occur in a context where the FASD (likely even the potential role of prenatal alcohol exposure) is not routinely considered [25]. In previous reports we have suggested that a context of prenatal alcohol exposure should be considered in all infant and childhood deaths [25]. A diagnosis of FASD is associated with increased risk of death for affected people and their siblings (even when the sibling FASD status is unknown) [21] [22]. In populations of children with an FASD, recent reports suggests a 5\% - 6\% mortality risk [10] [21] [22] [26]. Several reports have also demonstrated increased rates of mortality in mothers of children with FAS [10] [27]-[30].

We present several strategies which might improve the ascertainment of FASD in mortality events. 1) Larger population studies with improved exposure assessments. 2) Studies to develop strategies to routinely configure FASD and PAE into the decisional algorithm for classification of cause of death. 3) Development of risk stratification strategies to identify deaths at risk for having an FASD. Estimation of risk would be improved by a careful review of relevant data from the mothers and child's records. These would include use of information of previous maternal substance abuse treatment of all types (alcohol is often used in combination with other drugs), review of prenatal care records, identification of prenatal alcohol exposure, exposure dosimetry, maternal death, sibling with a diagnosis of FASD, sibling death, and adoption or placement in foster care.

The context of prenatal alcohol exposure and a diagnosis of FASD can offer important information on a va- 
riety of multifaceted and interacting risk markers and increase our understanding of the risk for mortality and adverse outcomes. This schema could be considered analogous to the concept of an infant of a diabetic mother where the infants are considered candidates for increased surveillance for a wide range of adverse outcomes. It is also important to clarify that the diagnosis of a chromosomal disorder, genetic abnormality, birth defect, infection, or traumatic cause of death does not exclude alcohol as an important consideration in risk assessment for mortality and other adverse events.

In previous research we have described the mechanisms of expression of neurobehavioral deficits in FASD [13]. Prenatal alcohol exposure modifies risk and phenotype severity by three primary pathways: 1) by lowering thresholds for expression of neurobehavioral deficits; 2) by increasing the number of comorbidities; 3) direct causation of some deficits. The structural deficits associated with prenatal alcohol exposure associated with mortality are large and include effects from exposure over multiple embryonic periods of development likely in concert with other modifiers of exposure. Importantly, non-lethal abnormalities are likely to have implications for subsequent neurobehavioral development and risk for mental disorders. These risks will likely change over time in response to past development and both age and development dependent demands. The potential range of neurobehavioral deficits seems large and in contrast to the typical facial features of FASD (thin upper lip, upturned nose, flat philtrim) many will have significant developmental implications far into the future as neurobehavioral development continues.

\section{Limitations}

Several important limitations for diagnosis of FASD are important in interpretation of this study. Firstly, the diagnostic nomenclature has been modified over the 35 year span of time (1974 to 2009) for cases included in this review [6] [31] [32]. Secondly, there is no widely accepted threshold for exposure that is necessary or sufficient to cause an FASD [33]. Thirdly, it is likely that many deaths occur in people with an FASD that is undiagnosed or where a past diagnosis is not available at the time of the death. It seems likely that these 57 cases represent a modest fraction of deaths in people with an FASD. Thus, the cause of death rankings may be incorrect.

Multicenter studies of mortality will likely be required to determine the extent of mortality risk in FASD and to identify the most prevalent causes of mortality. In the United States alone, there are 40,000 new cases of FASD each year and in the population birth through 18 years of age, about 720,000 people have an FASD. In this population several hundred deaths would be expected each year. This suggests that most cases of FASD are missed in mortality reviews. FASD should be considered as a risk marker for all-cause mortality in all family members (except fathers, where no mortality data are currently available).

\section{References}

[1] Sullivan, W.C. (2011) A Note on the Influence of maternal Inebriety on the Offspring.1899. International Journal of Epidemiology, 40, 278-282.

[2] Lemoine, P., Haroosseau, H., Borteryu, J.P. and Menuet, J.C. (1968) Les Enfants de Parents Alcooliques. Anomalies Observee a Propos de 127 cas [The Children of Alcoholic Parents: Anomalies Observed in 127 Cases] (in French). Quest Medicale, 21, 476-482.

[3] Lim, S.S., Vos, T., Flaxman, A.D., Danaei, G., Shibuya, K., Adair-Rohani, H., et al. (2012) A Comparative Risk Assessment of Burden of Disease and Injury Attributable to 67 Risk Factors and Risk Factor Clusters in 21 Regions, 1990-2010: A Systematic Analysis for the Global Burden of Disease Study. Lancet, 380, 2224-2260. http://dx.doi.org/10.1016/S0140-6736(12)61766-8

[4] Centers for Disease Control and Prevention (CDC) (2002) Alcohol Use among Women of Childbearing Age-United States, 1991-1999. MMWR Morbidity and Mortality Weekly Report, 51, 273-276.

[5] Centers for Disease Control and Prevention (CDC) (2009) Alcohol Use among Pregnant and Nonpregnant Women of Childbearing Age-United States, 1991-2005. MMWR Morbidity and Mortality Weekly Report, 58, 529-32.

[6] Stratton, K.R., Howe, C.J., Battaglia, F.C. (1996) Fetal Alcohol Syndrome-Diagnosis, Epidemiology, Prevention, and Treatment. National Academy Press, Washington DC.

[7] Morse, B., Gehshan, S. and Hutchins, E. (1997) Screening for Substance Abuse during Pregnancy: Improving Care, Improving Health. National Center for Education in Maternal and Child Health, Arlington.

[8] May, P.A., Gossage, J.P., Kalberg, W.O., Robinson, L.K., Buckley, D., Manning, M., et al. (2009) Prevalence and Epidemiologic Characteristics of FASD from Various Research Methods with an Emphasis on Recent In-School Studies. 
Developmental Disabilities Research Reviews, 15, 176-192. http://dx.doi.org/10.1002/ddrr.68

[9] Hofer, R. and Burd, L.J. (2009) Review of Published Studies of Kidney, Liver, and Gastrointestinal Birth Defects in Fetal Alcohol Spectrum Disorders. Birth Defects Research Part A-Clinical and Molecular Teratology, 85, 179-183. http://dx.doi.org/10.1002/bdra.20562

[10] Burd, L.J., Martsolf, J.T. and Klug, M.G. (1996) Children with Fetal Alcohol Syndrome in North Dakota: A Case Control Study Utilizing Birth Certificate Data. Addiction Biology, 1, 181-189. http://dx.doi.org/10.1080/1355621961000124806

[11] Abel, E.L. (1998) Fetal Alcohol Abuse Syndrome. Plenum Press, New York. http://dx.doi.org/10.1007/978-1-4757-5217-5

[12] Bagheri, M.M., Burd, L.J., Martsolf, J.T. and Klug, M.G. (1998) Fetal Alcohol Syndrome: Maternal and Neonatal Characteristics. Journal of Perinatal Medicine, 26, 263-269. http://dx.doi.org/10.1515/jpme.1998.26.4.263

[13] Burd, L.J., Klug, M.G., Martsolf, J. and Kerbeshian, J. (2003) Fetal Alcohol Syndrome: Neuropsychiatric Phenomics. Neurotoxicology and Teratology, 25, 697-705. http://dx.doi.org/10.1016/j.ntt.2003.07.014

[14] Patel, S.S., Burns, T.L., Botto, L.D., Riehle-Colarusso, T.J., Lin, A.E., Shaw, G.M., et al. (2012) Analysis of Selected Maternal Exposures and Non-Syndromic Atrioventricular Septal Defects in the National Birth Defects Prevention Study, 1997-2005. American Journal of Medical Genetics Part A, 158A, 2447-2455. http://dx.doi.org/10.1002/ajmg.a.35555

[15] Burd, L.J., Deal, E., Rios, R., Adickes, E., Wynne, J. and Klug, M.G. (2007) Congenital Heart Defects and Fetal Alcohol Spectrum Disorders. Congenital Heart Disease, 2, 250-255. http://dx.doi.org/10.1111/j.1747-0803.2007.00105.X

[16] Paintner, A., Williams, A.D. and Burd, L. (2012) Fetal Alcohol Spectrum Disorders-Implications for Child Neurology, Part 1: Prenatal Exposure and Dosimetry. Journal of Child Neurology, 27, 258-263. http://dx.doi.org/10.1177/0883073811428376

[17] Paintner, A., Williams, A.D and Burd, L. (2012) Fetal Alcohol Spectrum Disorders_-Implications for Child Neurology, Part 2: Diagnosis and Management. Journal of Child Neurology, 27, 355-362. http://dx.doi.org/10.1177/0883073811428377

[18] Gauthier, T.W., Drews-Botsch, C., Falek, A., Coles, C. and Brown, L.A. (2005) Maternal Alcohol Abuse and Neonatal Infection. Alcoholism: Clinical and Experimental Research, 29, 1035-1043. http://dx.doi.org/10.1097/01.ALC.0000167956.28160.5E

[19] Johnson, S., Knight, R., Marmer, D.J. and Steele, R.W. (1981) Immune Deficiency in Fetal Alcohol Syndrome. Pediatric Research, 15, 908-911. http://dx.doi.org/10.1203/00006450-198106000-00005

[20] Burd, L.J., Martsolf, J., Klug, M.G., O’Connor, E. and Peterson, M. (2003) Prenatal Alcohol Exposure Assessment: Multiple Embedded Measures in a Prenatal Questionnaire. Neurotoxicology and Teratology, 25, 675-679. http://dx.doi.org/10.1016/j.ntt.2003.07.019

[21] Burd, L.J., Klug, M.G. and Martsolf, J. (2004) Increased Sibling Mortality in Children with Fetal Alcohol Syndrome. Addiction Biology, 9,179-186. http://dx.doi.org/10.1080/13556210410001717088

[22] Burd, L.J., Klug, M.G., Bueling, R., Martsolf, J., Olson, M. and Kerbeshian, J. (2008) Mortality Rates in Subjects with Fetal Alcohol Spectrum Disorders and Their Siblings. Birth Defects Research Part A-Clinical and Molecular Teratology, 82, 217-223. http://dx.doi.org/10.1002/bdra.20445

[23] Qazi, Q., Masakawa, A., Milman, D., McGann, B., Chua, A. and Haller, J. (1979) Renal Anomalies in Fetal Alcohol Syndrome. Pediatrics, 63, 886-889.

[24] Taylor, C.L., Jones, K.L., Jones, M.C. and Kaplan, G.W. (1994) Incidence of Renal Anomalies in Children Prenatally Exposed to Ethanol. Pediatrics, 94, 209-212.

[25] Burd, L.J. and Wilson, H. (2004) Fetal, Infant, and Child Mortality in a Context of Alcohol Use. American Journal of Medical Genetics. Part C, Seminars in Medical Genetics, 127, 51-58. http://dx.doi.org/10.1002/ajmg.c.30016

[26] Habbick, B.F., Nanson, J.L., Snyder, R.E. and Casey, R.E. (1997) Mortality in Fetal Alcohol Syndrome. Canadian Journal of Public Health, 88, 181-183.

[27] Olegard, R., Sabel, K.G., Aronsson, M., Sandin, B., Johansson, P.R., Carlsson, C., et al. (1979) Effects on the Child of Alcohol Abuse during Pregnancy. Retrospective and Prospective Studies. Acta paediatrica Scandinavica Supplement, 275, 112-121. http://dx.doi.org/10.1111/j.1651-2227.1979.tb06170.x

[28] Streissguth, A.P., Clarren, S.K. and Jones, K.L. (1985) Natural History of the Fetal Alcohol Syndrome: A 10-Year Follow-Up of Eleven Patients. Lancet, 2, 85-91. http://dx.doi.org/10.1016/S0140-6736(85)90189-8

[29] Astley, S.J., Bailey, D., Talbot, C. and Clarren, S.K. (2000) Fetal Alcohol Syndrome (FAS) Primary Prevention through FAS Diagnosis: II. A Comprehensive Profile of 80 Birth Mothers of Children with FAS. Alcohol and Alcohol- 
ism, 35, 509-519. http://dx.doi.org/10.1093/alcalc/35.5.509

[30] Li, Q., Fisher, W.W., Peng, C.Z., Williams, A.D. and Burd, L. (2012) Fetal Alcohol Spectrum Disorders: A Population Based Study of Premature Mortality Rates in the Mothers. Maternal and Child Health Journal, 16, 1332-1337. http://dx.doi.org/10.1007/s10995-011-0844-3

[31] Jones, K.L. and Smith, D.W. (1975) The Fetal Alcohol Syndrome. Teratology, 12, 1-10. http://dx.doi.org/10.1002/tera.1420120102

[32] National Institute on Alcohol Abuse and Alcoholism (2011) Consensus Statement: Recognizing Alcohol-Related Neurodevelopmental Disorder (ARND) in Primary Health Care of Children. http://www.niaaa.nih.gov/sites/default/files/ARNDConferenceConsensusStatementBooklet_Complete.pdf .

[33] Burd, L., Blair, J. and Dropps, K. (2012) Prenatal Alcohol Exposure, Blood Alcohol Concentrations and Alcohol Elimination Rates for the Mother, Fetus and Newborn. Journal of Perinatology, 32, 652-659. http://dx.doi.org/10.1038/jp.2012.57

[34] van Rensburg, L.J. (1981) Major skeletal defects in the fetal alcohol syndrome. A case report. South African Medical Journal, 59, 687-688.

[35] Kinney, H., Faix, R. and Brazy, J. (1980) The fetal alcohol syndrome and neuroblastoma. Pediatrics, 66, 130-132.

[36] Beattie, J.O., Day, R.E., Cockburn, F. and Garg, R.A. (1983) Alcohol and the Fetus in the West of Scotland. British Medical Journal (Clinical Research Ed.), 287, 17-20. http://dx.doi.org/10.1136/bmj.287.6384.17

[37] Wisniewski, K., Dambska, M., Sher, J.H. and Qazi, Q. (1983) A Clinical Neuropathological Study of the Fetal Alcohol Syndrome. Neuropediatrics, 14, 197-201. http://dx.doi.org/10.1055/s-2008-1059578

[38] Peiffer, J., Majewski, F., Fischbach, H., Bierich, J.R. and Volk, B. (1979) Alcohol Embryo- and Fetopathy. Neuropathology of 3 Children and 3 Fetuses. Journal of the Neurological Sciences, 41, 125-137. http://dx.doi.org/10.1016/0022-510X(79)90033-9

[39] Coulter, C.L., Leech, R.W., Schaefer, G.B., Scheithauer, B.W. and Brumback, R.A. (1993) Midline Cerebral Dysgenesis, Dysfunction of the Hypothalamic-Pituitary Axis, and Fetal Alcohol Effects. Archives of Neurology, 50, 771-775. http://dx.doi.org/10.1001/archneur.1993.00540070083022

[40] Clarren, S.K., Alvord Jr., E.C., Sumi, S.M., Streissguth, A.P. and Smith, D.W. (1978) Brain Malformations Related to Prenatal Exposure to Ethanol. Journal of Pediatrics, 92, 64-67. http://dx.doi.org/10.1016/S0022-3476(78)80072-9

[41] Majewski, F. (1981) Alcohol Embryopathy: Some Facts and Speculations about Pathogenesis. Neurobehavioral Toxicology and Teratology, 3, 129-144.

[42] Terrapon, M., Schneider, P., Friedli, B. and Cox, J.N. (1977) Aortic Arch Interruption Type A with Aortopulmonary Fenestration in an Offspring of a Chronic Alcoholic Mother ("Fetal Alcohol Syndrome"). Helvetica Paediatrica Acta, 32, 141-148.

[43] Sonada, T., Ohdo, S., Sennari, E. and Madokoro, H. (1984) Congenital Heart Disease in a Case with Fetal Alcohol Syndrome: Clinical and Postmortem Findings. Pediatrics International, 26, 241-247. http://dx.doi.org/10.1111/j.1442-200X.1984.tb01820.x

[44] Ferrer, I. and Galofre, E. (1987) Dendritic Spine Anomalies in Fetal Alcohol Syndrome. Neuropediatrics, 18, $161-163$. http://dx.doi.org/10.1055/s-2008-1052472

[45] Lodha, A.K .and Satodia, P., Whyte, H. (2005) Fetal Alcohol Syndrome and Pyloric Stenosis: Alcohol Induced or an Association? Journal of Perinatal Medicine, 33, 262-263. http://dx.doi.org/10.1515/JPM.2005.049

[46] Dambska, M., Wisniewski, K.E. and Sher, J.H. (1986) Marginal Glioneuronal Heterotopias in Nine Cases with and without Cortical Abnormalities. Journal of Child Neurology, 1, 149-157. http://dx.doi.org/10.1177/088307388600100211

[47] Su, P.H., Chen, J.Y., Lee, I.C., Ng, Y.Y., Hu, J.M. and Chen, S.J. (2009) Pfeiffer-Like Syndrome with Holoprosencephaly: A Newborn with Maternal Smoking and Alcohol Exposure. Pediatrics \& Neonatology, 50, 234-238. http://dx.doi.org/10.1016/S1875-9572(09)60069-3

[48] Loser, H. and Majewski, F. (1977) Type and Frequency of Cardiac Defects in Embryofetal Alcohol Syndrome. Report of 16 Cases. British Heart Journal, 39, 1374-1379. http://dx.doi.org/10.1136/hrt.39.12.1374

[49] Adebahr, G. and Erkrath, K.D. (1984) Supernumerary Mammillary Bodies—Findings in a Case of Fetal Alcohol Syndrome. Zeitschrift fur Rechtsmedizin, 92, 239-246.

[50] Manzke, H. and Grosse, F.R. (1975) Incomplete and Complete "Fetal Alcohol Syndrome” in Three Children of an Alcoholic Mother. Die Medizinische Welt, 26, 709-712.

[51] Khan, A., Bader, J.L., Hoy, G.R. and Sinks, L.F. (1979) Hepatoblastoma in Child with Fetal Alcohol Syndrome. Lancet, 1, 1403-1074. http://dx.doi.org/10.1016/S0140-6736(79)92035-X 
[52] Ronen, G.M. and Andrews, W.L. (1991) Holoprosencephaly as a Possible Embryonic Alcohol Effect. American Journal of Medical Genetics, 40, 151-154. http://dx.doi.org/10.1002/ajmg.1320400206

[53] Becker, H., Zaunschirm, A., Muntean, W. and Domej, W. (1982) Fetal Alcohol Syndrome and Malignant Tumors. Wiener klinische Wochenschrift, 94, 364-365.

[54] Hornstein, L., Crowe, C. and Gruppo, R. (1977) Adrenal Carcinoma in Child with History of Fetal Alcohol Syndrome. Lancet, 2, 1292-1293. http://dx.doi.org/10.1016/S0140-6736(77)92706-4

[55] Zaunschirm, A. and Muntean, W. (1984) Fetal Alcohol Syndrome and Malignant Disease. European Journal of Pediatrics, 141, 256. http://dx.doi.org/10.1007/BF00572775 